\title{
Prognostic Value of Lymphovascular Invasion in Node-Negative Upper Urinary Tract Urothelial Carcinoma Patients Undergoing Radical Nephroureterectomy
}

\author{
Wen Liu, Zhonghan Zhou, Dahai Dong, Lijiang Sun, and Guiming Zhang \\ Department of Urology, The Affiliated Hospital of Qingdao University, Qingdao, China.
}

Purpose: This study aimed to evaluate the prognostic impact of lymphovascular invasion (LVI) in patients treated with radical nephroureterectomy (RNU) for upper urinary tract urothelial carcinoma (UTUC).

Materials and Methods: We collected data from 180 patients who were treated with RNU from 2005 to 2013 at our institution. The Kaplan-Meier method with log-rank test and Cox proportional hazards regression models were used for univariate and multivariate analyses.

Results: LVI was present in 28 patients (15.6\%), which was associated with higher pathological tumor stage ( $p<0.001)$, tumor necrosis ( $p=0.012)$, lymph node metastasis $(p=0.017)$ and multifocality $(p=0.012)$. On multivariate analysis, LVI was an independent prognostic factor of recurrence-free survival [RFS: hazard ratio (HR) $=2.954 ; 95 \%$ confidence interval $(\mathrm{CI})=1.539-5.671 ; p=0.001$ ] and cancer-specific survival (CSS: HR=3.530; 95\% CI=1.701-7.325; $p=0.001$ ) in all patients. In patients with node-negative UTUC, LVI was also a significant predictor of RFS (HR=3.732; 95\% CI 1.866-7.464; $p<0.001)$ and CSS (HR=3.825; 95\% CI=1.777-8.234; $p=0.001$ ).

Conclusion: LVI status was an independent predictor in patients with UTUC who underwent RNU. The estimate of LVI could help physicians identify high-risk patients and make a better medication regimen of adjuvant chemotherapy.

Key Words: Urinary tract; prognosis; neoplasm invasiveness; carcinoma, transitional cell; lymph nodes

\section{INTRODUCTION}

Upper urinary tract urothelial carcinoma (UTUC), which originates from the urothelial lining, can be located in pyelocaliceal cavities and ureter. The incidence of UTUC is much less than that of bladder cancer, accounting for $10 \%$ of renal tumors and $5 \%$ of all urothelial malignancies. ${ }^{1-3}$ The standard opera-

\footnotetext{
Received: August 16, 2018 Revised: October 24, 2018

Accepted: November 25, 2018

Corresponding author: Guiming Zhang, PhD, Department of Urology, The Affiliated Hospital of Qingdao University, No. 16, Jiangsu Road, Qingdao 266003, China. Tel: 86-532-8291-3056, Fax: 86-532-8291-1999, E-mail: zhangguiming9@126.com

-The authors have no potential conflicts of interest to disclose.

(c) Copyright: Yonsei University College of Medicine 2019

This is an Open Access article distributed under the terms of the Creative Commons Attribution Non-Commercial License (https://creativecommons.org/licenses/ by-nc/4.0) which permits unrestricted non-commercial use, distribution, and reproduction in any medium, provided the original work is properly cited.
}

tion method for UTUC is radical nephroureterectomy (RNU) with removing bladder cuff, regardless of high-risk noninvasive and invasive UTUC. ${ }^{4,5}$ However, invasive UTUC accounts for approximately $60 \%$ of total cases, which is much higher than the invasive percentage of bladder cancer. ${ }^{5}$ The outcome is still commonly poor with a high recurrence rate ranging from $30 \%$ to $75 \%$ in UTUC. ${ }^{2,6}$ Several studies have shown that patient's age, history of bladder cancer, tumor stage, tumor grade, lymph node metastasis, necrosis, multifocality, and hydronephrosis are independent prognostic factors connected to disease recurrence or survival. ${ }^{1,6-8}$

Lymphovascular invasion (LVI) is a significant and crucial step in the systemic dissemination of cancer cells. ${ }^{9}$ LVI has also been discovered as a prognostic factor in UTUC. ${ }^{10-14}$ However, the influence of LVI on prognosis was meaningless in some studies, ${ }^{15-17}$ which remains a controversial problem. In pathological reports, LVI was routinely assessed but not docu- 
mented in all cases, and not described as a prognostic factor. ${ }^{10}$ Since LVI predicted that lymph node metastasis was a prognostic factor in UTUC patients, ${ }^{18,19}$ focusing on node-negative patients might have great significance in discovering high-risk factors in patients with node-negative UTUC. In the present study, we aimed to further validate the prognostic value of LVI in patients with UTUC, especially in those with node-negative UTUC undergoing RNU. Improved understanding of prognostic influence of LVI can help us make better prognostic evaluations and more appropriate regimen of adjuvant chemotherapy.

\section{MATERIALS AND METHODS}

\section{Ethical approval}

The study protocol was approved by ethics committee at our institution. All patients involved in this retrospective study have given written informed consent. We made sure that all samples were anonymous.

\section{Patient selection and follow-up}

From 2005 to 2013, 180 patients, who were diagnosed with UTUC and treated with RNU in our institution, were analyzed retrospectively. When lymph node enlargement was doubtable on preoperative CT or enlarged during intraoperative examinations, regional lymphadenectomy was routinely performed without extending lymphadenectomy. Patients with incomplete clinical data, non-UTUC, or distant metastasis were excluded. Finally, 180 patients were enrolled in this study.

The study end was recurrence-free survival (RFS) and cancer-specific survival (CSS) after RNU. Disease RFS was defined as local recurrence and lymph node and/or distant metastasis, except the contralateral upper urinary tract or subsequent bladder recurrences. We defined CSS according to death certificates and results estimated by treating physicians. However, patients who died within 30 days of surgery or before discharge were not included as disease recurrence and cancer-specific death cases.

\section{Pathologic evaluation}

All specimens were re-reviewed by two genitourinary pathologists according to standard pathologic procedures, to ensure the validity of pathologic data extraction. Tumor stage was assessed according to the 2018 American Joint Committee on Cancer (AJCC) TNM staging system. Tumor grade was evaluated according to the 2016 World Health Organization grading system. ${ }^{20}$ The clinicopathological data of patients included age, history of bladder cancer, tumor stage, tumor grade, lymph node involvement, necrosis, multifocality, and hydronephrosis.

Hematoxylin and eosin (H\&E) staining is regularly performed in our institute to identify LVI, which is defined pathologically as the presence of tumor cells within a definite endotheliumlined space without underlying muscular walls both in the intratumoral and peritumoral area, or spaces in vascular lined by plump cells at the peritumoral area (Fig. 1A). ${ }^{21}$ Erythrocytes or fibrin clots in blood vessels, and the lack of smooth muscle or elastic fibers in lymphatic vessels can help pathologists to identify vessels and LVI. Immunohistochemical (IHC) staining is not routinely used to assess LVI, unless two pathologists cannot reach an agreement on the confirmation of LVI in one slide (Fig. 1B). However, it is difficult to distinguish between vascular and lymphatic invasion due to the difficulty and the lack of reproducibility when using routine light microscopic examinations. Nodal status was assessed by pathologists when lymph nodes were retrieved during operation.

\section{Statistical analysis}

Statistical analyses were performed by SPSS v.16.0 (SPSS Inc., Chicago, IL, USA). The correlation between LVI and other clinicopathological characteristics was tested by $\chi^{2}$ test or Fisher's exact test. Survival curve for the presence of LVI was estimated with Kaplan-Meier method, and differences were assessed by log-rank statistic. Univariate and multivariate regression analyses were performed with Cox proportional hazards regression model to explain the impact of LVI on RFS and CSS
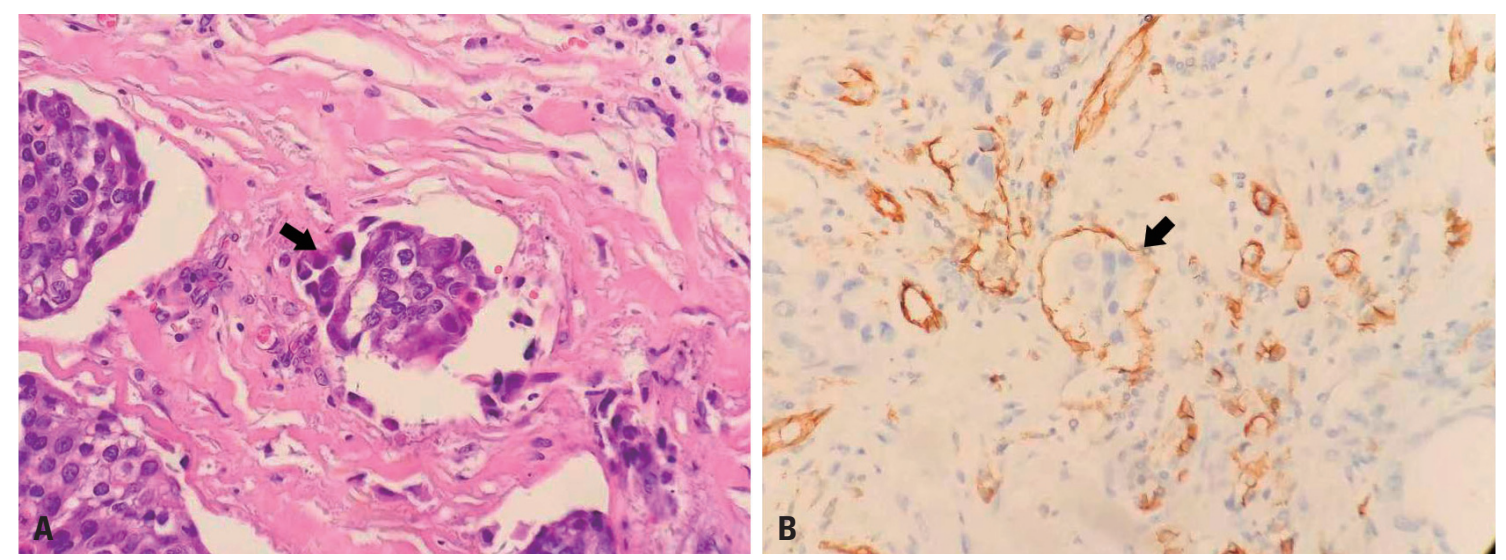

Fig. 1. Lymphovascular invasion (arrow) in upper urinary tract urothelial carcinoma was shown in hematoxylin and eosin staining (A) and immunohistochemical staining (CD31) (B); $\times 400$. 
respectively, or in patients without lymph nodes involvement. The results showed that hazard ratios (HRs) served as relative risks with corresponding 95\% confidence intervals (CIs). All reported $p$ values were two-sided, and statistical significance was set at $p \leq 0.05$.

\section{RESULTS}

\section{Patient characteristics}

Our study included 180 patients with UTUC, consisting of 109 males (60.6\%) and 71 females (39.4\%). Clinicopathological characteristics are listed in Table 1 . The average age of these 180 patients was 67.2 years (ranging from 39 to 87 years), and the mean follow-up period was 45.4 months (ranging from 3 to 180 months).

\section{Association of LVI with clinicopathological features}

LVI, present in 28 patients (15.6\%), was significantly associated with advanced pathological stage $(p<0.001)$, tumor necrosis ( $p=0.012)$, lymph nodes metastasis $(p=0.017)$, and multifocality ( $p=0.012$ ) (Table 1$)$. Exclusion of lymph node-positive patients did not alter the significant correlation of LVI with advanced pathological stage $(p=0.008)$, tumor necrosis $(p=$ $0.007)$, and multifocality ( $p=0.007$ ) (data not shown).

\section{Correlation of LVI with clinical prognosis in all patients} LVI was closely associated with RFS and CSS (Tables 2 and 3). During follow-up, 39 patients (21.7\%) experienced disease recurrence and 31 patients (17.2\%) died of UTUC. The 5-year RFS rate was $84.8 \%$ in the absence of LVI and $35.0 \%$ in the presence of LVI ( $p<0.001$ ) (Fig. 2A). The 5 -year CSS rate was $87.5 \%$ in the absence of LVI and $25.2 \%$ in the presence of LVI ( $p<$ 0.001) (Fig. 2B). Univariate analysis revealed that LVI were as-

Table 1. Association of Lymphovascular Invasion with Clinical and Pathologic Characteristics of Patients Treated with Radical Nephroureterectomy for Upper Urinary Tract Urothelial Carcinoma

\begin{tabular}{|c|c|c|c|c|}
\hline & \multirow{2}{*}{ Cases (\%) } & \multicolumn{3}{|c|}{ Lymphovascular invasion } \\
\hline & & Absent (\%) (n=152, 84.4\%) & Present $(\%)(n=28,15.6 \%)$ & $p$ value \\
\hline Gender & & & & 0.214 \\
\hline Male & $109(60.6)$ & $95(62.5)$ & $14(50.0)$ & \\
\hline Female & $71(39.4)$ & $57(37.5)$ & $14(50.0)$ & \\
\hline Age (yr) & & & & 0.563 \\
\hline$<70$ & $99(55.0)$ & $85(55.9)$ & $14(50.0)$ & \\
\hline$\geq 70$ & $81(45.0)$ & $67(44.1)$ & $14(50.0)$ & \\
\hline Smoking & & & & 0.076 \\
\hline Yes & $52(28.9)$ & $40(26.3)$ & $12(42.9)$ & \\
\hline No & $128(71.1)$ & $112(87.5)$ & $16(57.1)$ & \\
\hline History of bladder cancer & & & & 0.994 \\
\hline No & $164(91.1)$ & $139(91.4)$ & $25(89.3)$ & \\
\hline Yes & $16(8.9)$ & $13(8.6)$ & $3(10.7)$ & \\
\hline Pathologic tumor stage & & & & 0.001 \\
\hline Ta-T2 & $115(63.9)$ & $105(69.1)$ & $10(35.7)$ & \\
\hline T3-T4 & $65(36.1)$ & 47 (30.9) & $18(64.3)$ & \\
\hline Tumor grade & & & & 0.194 \\
\hline G1-G2 & $91(50.6)$ & $80(52.6)$ & $11(39.3)$ & \\
\hline G3 & $89(49.4)$ & $72(47.4)$ & $17(60.7)$ & \\
\hline Tumor necrosis* & & & & 0.012 \\
\hline Absent & $173(96.1)$ & $149(98.0)$ & $24(85.7)$ & \\
\hline Present & $7(3.9)$ & $3(2.0)$ & $4(14.3)$ & \\
\hline Multifocality* & & & & 0.012 \\
\hline Unifocal & $173(96.1)$ & $149(98.0)$ & $24(85.7)$ & \\
\hline Multifocal & $7(3.9)$ & $3(2.0)$ & $4(14.3)$ & \\
\hline Lymph node metastasis & & & & 0.017 \\
\hline Absent & $169(93.9)$ & $146(96.1)$ & $23(82.1)$ & \\
\hline Present & $11(6.1)$ & $6(3.9)$ & $5(17.9)$ & \\
\hline Hydronephrosis & & & & 0.737 \\
\hline Absent & $72(40.0)$ & $60(39.5)$ & $12(42.9)$ & \\
\hline Present & $108(60.0)$ & $92(60.5)$ & $16(57.1)$ & \\
\hline
\end{tabular}

*Fisher's exact test. 
sociated with RFS and CSS (double $p<0.001$ ). After adjusting for the impact of factors including patient's age, tumor stage, tumor grade, lymph node metastasis, and multifocality, multivariate analysis suggested that LVI was an independent prognostic factor of RFS (HR=2.954; 95\% CI=1.539-5.671; $p=0.001)$ (Table 2) and CSS (HR=3.530; 95\% CI=1.701-7.325; $p=0.001$ )
(Table 3).

\section{Correlation of LVI with clinical prognosis in node-negative patients}

The number of patients with node-negative UTUC was 169. In patients without lymph node involvement, the 5 year RFS and

Table 2. Univariate and Multivariate Cox Regression Analyses of All Patients for Disease Recurrence-Free Survival

\begin{tabular}{|c|c|c|c|c|}
\hline \multirow{2}{*}{ Variable } & \multicolumn{2}{|c|}{ Univariate analysis } & \multicolumn{2}{|c|}{ Multivariate analysis } \\
\hline & HR (95\% Cl) & $p$ value & HR $(95 \%$ CI) & $p$ value \\
\hline Gender & & 0.937 & & \\
\hline Age (yr) & $2.310(1.217-4.384)$ & 0.010 & & \\
\hline Smoking & 2.242 (1.186-4.239) & 0.013 & & \\
\hline History of bladder cancer & & 0.277 & & \\
\hline Pathologic tumor stage & 17.972 (7.389-43.496) & $<0.001$ & 13.688 (5.55-33.755) & $<0.001$ \\
\hline Grade & $2.637(1.334-5.211)$ & 0.005 & & \\
\hline Lymphovascular invasion & 6.007 (3.170-11.382) & $<0.001$ & $2.954(1.539-5.671)$ & 0.001 \\
\hline Tumor necrosis & & 0.103 & & \\
\hline Multifocality & 3.284 (1.008-10.696) & 0.048 & & \\
\hline Lymph node metastasis & & 0.749 & & \\
\hline Hydronephrosis & & 0.919 & & \\
\hline
\end{tabular}

$\mathrm{Cl}$, confidence interval; $\mathrm{HR}$, hazard ratio.

Table 3. Univariate and Multivariate Cox Regression Analyses of all Patients for Cancer-Specific Survival

\begin{tabular}{|c|c|c|c|c|}
\hline \multirow{2}{*}{ Variable } & \multicolumn{2}{|c|}{ Univariate analysis } & \multicolumn{2}{|c|}{ Multivariate analysis } \\
\hline & HR (95\% CI) & $p$ value & HR (95\% CI) & $p$ value \\
\hline Gender & & 0.912 & & \\
\hline Age (yr) & $3.498(1.643-7.448)$ & 0.001 & & \\
\hline Smoking & $2.683(1.323-5.441)$ & 0.006 & & \\
\hline History of bladder cancer & & 0.650 & & \\
\hline Pathologic tumor stage & 18.231 (6.357-52.284) & $<0.001$ & 12.782 (4.33-37.756) & $<0.001$ \\
\hline Grade & 2.206 (1.038-4.686) & 0.040 & & \\
\hline Lymphovascular invasion & 7.595 (3.722-15.495) & $<0.001$ & 3.530 (1.701-7.325) & 0.001 \\
\hline Tumor necrosis & & 0.053 & & \\
\hline Multifocality & $4.013(1.212-13.288)$ & 0.023 & & \\
\hline Lymph node metastasis & & 0.491 & & \\
\hline Hydronephrosis & & 0.880 & & \\
\hline
\end{tabular}

$\mathrm{Cl}$, confidence interval; $\mathrm{HR}$, hazard ratio.
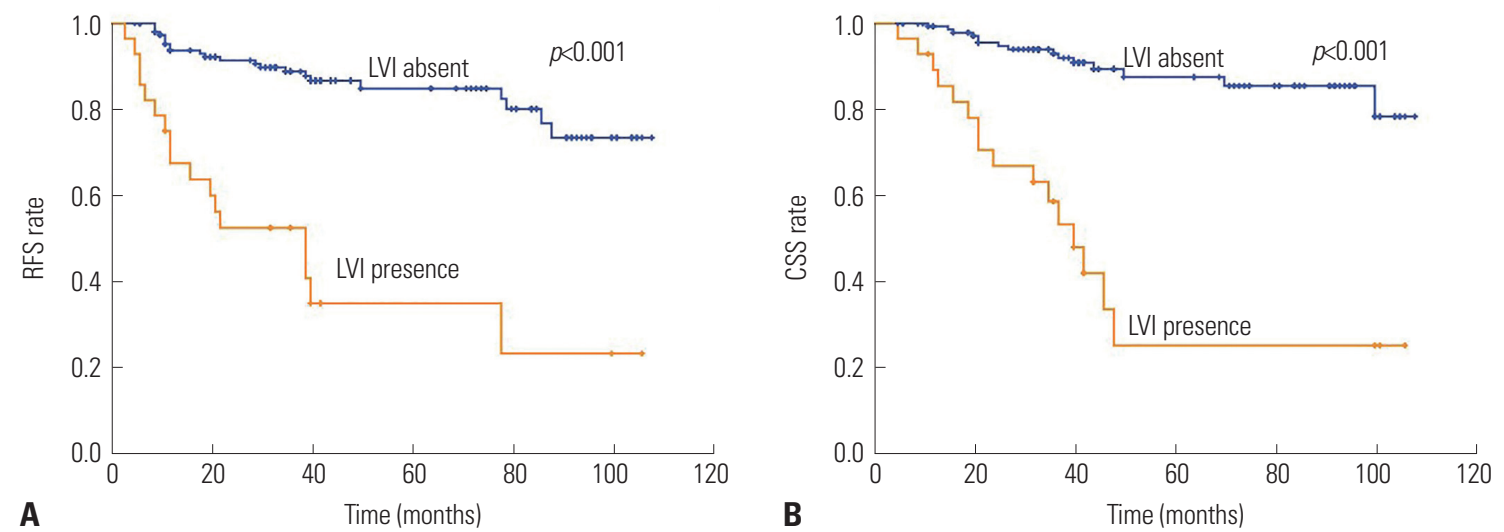

Fig. 2. Kaplan-Meier curves of disease RFS (A) and CSS (B) stratified by LVI in 180 patients with upper urinary tract urothelial carcinoma undergoing radical nephroureterectomy. RFS, recurrence-free survival; CSS, cancer-specific survival; LVI, Iymphovascular invasion. 
CSS rates were $84.1 \%$ and $86.9 \%$, respectively, in the absence of LVI, compared to $33.8 \%$ and $21.7 \%$, respectively, in the presence of LVI (double $p<0.001$ ) (Fig. 3). LVI was an independent prognostic factor of RFS (HR=3.732; 95\% CI 1.866-7.464; $p<0.001$ ) (Table 4) and CSS ( $\mathrm{HR}=3.825 ; 95 \% \mathrm{CI}=1.777-8.234$; $p=0.001$ ) (Table 5).

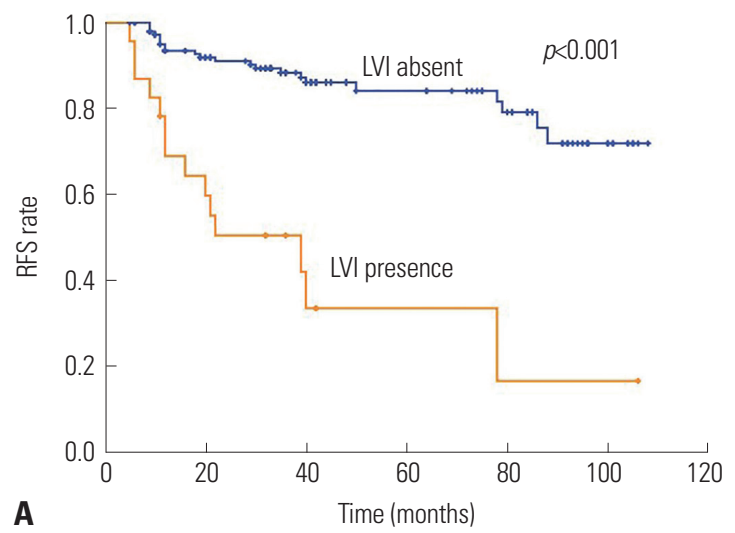

\section{DISCUSSION}

LVI has been proven to be a significant prognostic factor in various malignancies such as liver, testis, and penile cancer. ${ }^{12}$ Some studies have estimated that LVI was a poor prognostic predictor in patients with UTUC after RNU. ${ }^{10-13}$ However, the influence of LVI in prognosis was meaningless in other stud-

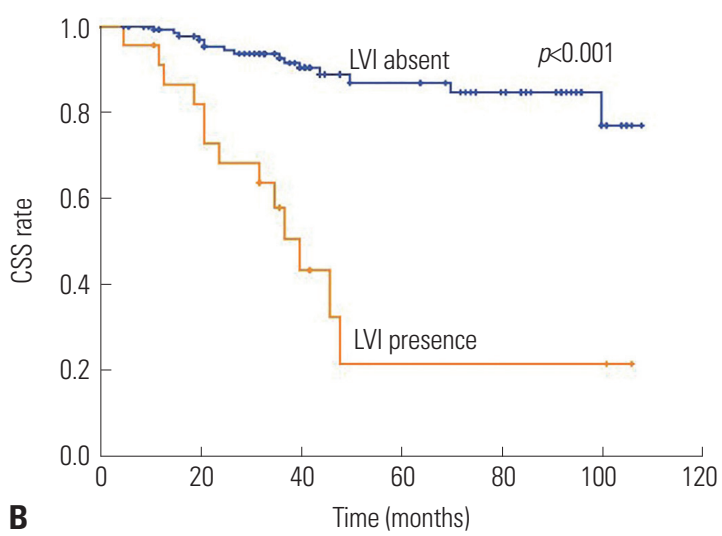

Fig. 3. Kaplan-Meier curves of disease RFS (A) and CSS (B) stratified by LVI in 169 patients with node-negative upper urinary tract urothelial carcinoma undergoing radical nephroureterectomy. RFS, recurrence-free survival; CSS, cancer-specific survival; LVI, lymphovascular invasion.

Table 4. Univariate and Multivariate Cox Regression Analyses of Node-Negative Patients for Disease Recurrence-Free Survival

\begin{tabular}{|c|c|c|c|c|}
\hline \multirow{2}{*}{ Variable } & \multicolumn{2}{|l|}{ Univariate analysis } & \multicolumn{2}{|l|}{ Multivariate analysis } \\
\hline & HR $(95 \%$ CI) & $p$ value & $\mathrm{HR}(95 \% \mathrm{CI})$ & $p$ value \\
\hline Gender & & 0.588 & & \\
\hline Age (yr) & $2.078(1.072-4.026)$ & 0.030 & & \\
\hline Smoking & & 0.099 & & \\
\hline History of bladder cancer & & 0.258 & & \\
\hline Pathologic tumor stage & 17.914 (7.199-44.581) & $<0.001$ & 15.269 (6.01-38.785) & $<0.001$ \\
\hline Grade & 2.624 (1.289-5.340) & 0.008 & & \\
\hline Lymphovascular invasion & 6.097 (3.082-12.063) & $<0.001$ & $3.732(1.866-7.464)$ & $<0.001$ \\
\hline Tumor necrosis & & 0.099 & & \\
\hline Multifocality & 3.400 (1.039-11.125) & 0.043 & & \\
\hline Hydronephrosis & & 0.860 & & \\
\hline
\end{tabular}

$\mathrm{Cl}$, confidence interval; $\mathrm{HR}$, hazard ratio.

Table 5. Univariate and Multivariate Cox Regression Analyses of Node-Negative Patients for Cancer-Specific Survival

\begin{tabular}{|c|c|c|c|c|}
\hline \multirow{2}{*}{ Variable } & \multicolumn{2}{|l|}{ Univariate analysis } & \multicolumn{2}{|l|}{ Multivariate analysis } \\
\hline & HR $(95 \%$ CI) & $p$ value & $\mathrm{HR}(95 \% \mathrm{CI})$ & $p$ value \\
\hline Gender & & 0.701 & & \\
\hline Age (yr) & $3.114(1.431-6.775)$ & 0.004 & & \\
\hline Smoking & & 0.055 & & \\
\hline History of bladder cancer & & 0.602 & & \\
\hline Pathologic tumor stage & 16.768 (5.786-48.593) & $<0.001$ & 12.414 (4.19-36.784) & $<0.001$ \\
\hline Grade & & 0.051 & & \\
\hline Lymphovascular invasion & 7.445 (3.500-15.835) & $<0.001$ & 3.825 (1.777-8.234) & 0.001 \\
\hline Tumor necrosis & & 0.058 & & \\
\hline Multifocality & $4.264(1.277-14.241)$ & 0.018 & & \\
\hline Hydronephrosis & & 0.648 & & \\
\hline
\end{tabular}

$\mathrm{Cl}$, confidence interval; $\mathrm{HR}$, hazard ratio. 
ies, ${ }^{15-17}$ which remains a controversial problem.

In consonance with previous studies, our results indicated that LVI was a poor independent predictor of RFS ( $p=0.001)$ and CSS $(p=0.001)$ in UTUC patients. ${ }^{10-13}$ Most node-positive patients with UTUC had early distant metastasis after RNU. ${ }^{19}$ Since lymph node involvement was thought as an adverse factor in UTUC, focusing on node-negative patients might have a great significance in discovering the high-risk factors in them. However, only a few studies analyzed the association between LVI and the outcome of patients with node-negative UTUC. In node-negative patients, multivariate analysis suggested that LVI was associated with poor prognosis in RFS $(p<0.001)$ and CSS ( $p=0.001$ ). Therefore, our study suggested that LVI was also a poor predictor of RFS and CSS in node-negative patients. On this basis, pathologic assessment of UTUC specimens should be routinely recorded for LVI, especially in node-negative ones. In addition, LVI was associated with established features of UTUC, such as advanced pathological tumor stage, presence of tumor necrosis, and multifocality, which are all independent poor prognostic factors. ${ }^{6-8}$ Nowadays, tumor stage is the most important prognostic indicator of UTUC. We acquired the same results that advanced pathological tumor stage was an important prognostic factor in RFS (HR=13.688; 95\% $\mathrm{CI}=5.55-33.755 ; p<0.001)$ and $\mathrm{CSS}(\mathrm{HR}=12.782 ; 95 \% \mathrm{CI}=$ 4.33-37.756; $p<0.001$ ), respectively.

LVI has been involved in TNM classification of some tumors, such as lung cancer, colorectal cancer, gastric cancer, and testicular cancer, ${ }^{22-25}$ which meant that LVI might have a similar significance in TNM classification. In non-small-cell lung cancer (NSCLC), stage IA with vascular invasion (VI) and stage IB had equivalent prognostic outcomes, suggesting that pT IA NSCLC with VI should be upstaged similar to pT IB NSCLC. ${ }^{22}$ Ahmed Farag, et al. ${ }^{23}$ found that adding LVI to TNM classification of colorectal cancer could help the physician provide more aggressive treatment strategies for patients with early TNM stage with LVI. In nonseminomatous germ cell tumor, stage I patients with LVI were advocated to accept adjuvant chemotherapy compared to patients without LVI advocated to accept surveillance. ${ }^{25}$ According to our results, LVI should be evaluated as reference criteria for high risk stratification and included in TNM classification system for UTUC, especially for patients with node-negative UTUC. Further prospective studies should focus more on the influence of LVI on UTUC patients of different TNM stages.

LVI was defined as the presence of tumor cells within an endothelium-lined space of vascular or lymphatic vessels without underlying muscular walls both in the intratumoral and peritumoral area, or spaces in vascular lined by plump cells at the peritumoral area. ${ }^{21}$ It is an important step in the mechanisms of tumor growth, lymph node involvement, and distant metastasis. There was no prognostic value of LVI in node-positive patients (data not shown) compared to node-negative ones. LVI and metastatic lymph nodes had a powerful associ- ation ( $p=0.017)$, which suggested that LVI might be an essential step for lymph node metastasis, and can prompt to seek for occult metastasis during the operation. Therefore, LVI can give a hint about whether or not regional lymph nodes should be removed. While H\&E staining has lower diagnostic accuracy in detecting LVI compared with using IHC staining, increasing application of IHC staining could reap more reliable result.

On account of the currently insufficient data of UTUC, the effect of adjuvant chemotherapy in UTUC still remains controversial. $^{26,27}$ Some studies showed that adjuvant chemotherapy improved CSS in patients with node-positive UTUC or positive LVI. ${ }^{28,29}$ Therefore, application of adjuvant chemotherapy should be personalized according to the situation of LVI or lymph node involvement. In node-negative patients with UTUC, the association of LVI with the effect of adjuvant chemotherapy has not been proven. Unfortunately, due to the limitation of sample size, we did not analyze the effect of adjuvant chemotherapy in patients with node-negative UTUC. While our results presented that LVI was a high-risk prognostic factor in node-negative patients with UTUC, it is probably an indicator guiding more accurate administration of adjuvant chemotherapy in node-negative patients.

There are several limitations in our study. First, this study had a single-center retrospective design, which carries an intrinsic bias. Further prospective study in a large-scale population should be performed to explore the prognostic implication of LVI in UTUC. Second, lymph node metastasis was proven as an outcome predictor in other results. ${ }^{11,13}$ We did not verify its prognostic role in our study. This was due to the small size of our enrolled patients, especially the node-positive patients. However, the prognostic implication of LVI in node-negative patients was more importantly and powerfully investigated in our study. Third, the criterion of LVI might have a bias due to the relatively lower accuracy compared to IHC staining. However, the application of IHC staining was limited on the account of its price and other reasons worldwide.

In summary, LVI is an independent adverse prognostic factor in patients with node-negative UTUC after RNU, which is linked to shorter RFS and CSS. LVI can be estimated as a selection criterion for high risk stratification, and help physicians make decisions on accurate administration of adjuvant chemotherapy. LVI should be routinely estimated in histological specimens.

\section{ACKNOWLEDGEMENTS}

This study was partly funded by the National Natural Science Foundation of China (Grant No. NSFC 81502195, NSFC 81672512) and the Medicine and Health Science Technology Development Project of Shandong Province (No. 2016WS0258). 


\section{AUTHOR CONTRIBUTIONS}

Conceptualization: Wen Liu, Guiming Zhang. Data curation: Wen Liu, Guiming Zhang. Formal analysis: Wen Liu, Lijiang Sun. Funding acquisition: Lijiang Sun, Guiming Zhang. Investigation: Wen Liu, Zhonghan Zhou, Dahai Dong. Methodology: Wen Liu, Zhonghan Zhou, Dahai Dong. Project administration: Lijiang Sun, Guiming Zhang. Resources: Guiming Zhang. Software: Wen Liu. Supervision: Lijiang Sun, Guiming Zhang. Validation: Wen Liu, Zhonghan Zhou. Visualization: Dahai Dong. Writing-original draft: Wen Liu. Writing-review and editing: Wen Liu, Guiming Zhang.

\section{ORCID iDs}

Wen Liu

Zhonghan Zhou

Dahai Dong

Lijiang Sun

Guiming Zhang https://orcid.org/0000-0002-9290-2217

https://orcid.org/0000-0002-7927-1393

https://orcid.org/0000-0001-6905-382X

https://orcid.org/0000-0001-5856-8270

https://orcid.org/0000-0002-5856-5325

\section{REFERENCES}

1. Rouprêt M, Babjuk M, Compérat E, Zigeuner R, Sylvester RJ, Burger M, et al. European Association of Urology Guidelines on Upper Urinary Tract Urothelial Carcinoma: 2017 update. Eur Urol 2018;73:111-22.

2. Siegel RL, Miller KD, Jemal A. Cancer statistics, 2017. CA Cancer J Clin 2017;67:7-30.

3. Munoz JJ, Ellison LM. Upper tract urothelial neoplasms: incidence and survival during the last 2 decades. J Urol 2000;164:1523-5.

4. Xylinas E, Rink M, Margulis V, Karakiewicz P, Novara G, Shariat SF; Upper Tract Urothelial Carcinoma Collaboration (UTUCC). Multifocal carcinoma in situ of the upper tract is associated with high risk of bladder cancer recurrence. Eur Urol 2012;61:1069-70.

5. Margulis V, Shariat SF, Matin SF, Kamat AM, Zigeuner R, Kikuchi E, et al. Outcomes of radical nephroureterectomy: a series from the Upper Tract Urothelial Carcinoma Collaboration. Cancer 2009; 115:1224-33.

6. Zhang XK, Zhang ZL, Yang P, Cai MY, Hu WM, Yun JP, et al. Tumor necrosis predicts poor clinical outcomes in patients with nodenegative upper urinary tract urothelial carcinoma. Jpn J Clin Oncol 2015;45:1069-75.

7. Morizane S, Iwamoto H, Masago T, Yao A, Isoyama T, Sejima T, et al. Preoperative prognostic factors after radical nephroureterectomy in patients with upper urinary tract urothelial carcinoma. Int Urol Nephrol 2013;45:99-106.

8. Verhoest G, Shariat SF, Chromecki TF, Raman JD, Margulis V, Novara $\mathrm{G}$, et al. Predictive factors of recurrence and survival of upper tract urothelial carcinomas. World J Urol 2011;29:495-501.

9. Sundar SS, Ganesan TS. Role of lymphangiogenesis in cancer. J Clin Oncol 2007;25:4298-307.

10. Mellouli M, Charfi S, Smaoui W, Kallel R, Khabir A, Bouacida M, et al. Prognostic role of lymphovascular invasion in patients with urothelial carcinoma of the upper urinary tract. Urol J 2017;14: 5008-12.

11. Lee HY, Li CC, Huang CN, Ke HL, Li WM, Liang PI, et al. Prognostic significance of lymphovascular invasion in upper urinary tract urothelial carcinoma is influenced by tumor location. Ann Surg Oncol 2015;22:1392-400.

12. Ku JH, Byun SS, Jeong H, Kwak C, Kim HH, Lee SE. Lymphovascular invasion as a prognostic factor in the upper urinary tract

urothelial carcinoma: a systematic review and meta-analysis. Eur J Cancer 2013;49:2665-80.

13. Novara G, Matsumoto K, Kassouf W, Walton TJ, Fritsche HM, Bastian PJ, et al. Prognostic role of lymphovascular invasion in patients with urothelial carcinoma of the upper urinary tract: an international validation study. Eur Urol 2010;57:1064-71.

14. Kikuchi E, Margulis V, Karakiewicz PI, Roscigno M, Mikami S, Lo$\tan \mathrm{Y}$, et al. Lymphovascular invasion predicts clinical outcomes in patients with node-negative upper tract urothelial carcinoma. J Clin Oncol 2009;27:612-8.

15. Yoshida T, Kinoshita H, Shimada S, Sugi M, Matsuda T. Preoperative pyuria is a poor prognostic factor in patients with urothelial carcinoma of the upper urinary tract after surgery. Clin Genitourin Cancer 2017;15:e543-50.

16. Mathieu R, Klatte T, Margulis V, Karam JA, Rouprêt M, Seitz C, et al. Survivin is not an independent prognostic factor for patients with upper tract urothelial carcinoma: a multi-institutional study. Urol Oncol 2015;33:495.e15-22.

17. Colin P, Ouzzane A, Yates DR, Audenet F, Pignot G, Arvin-Berod $A$, et al. Influence of positive surgical margin status after radical nephroureterectomy on upper urinary tract urothelial carcinoma survival. Ann Surg Oncol 2012;19:3613-20.

18. Raica M, Ribatti D. Targeting tumor lymphangiogenesis: an update. Curr Med Chem 2010;17:698-708.

19. Yuan X, Zhu C, Wang M, Mo F, Du W, Ma X. Night shift work increases the risks of multiple primary cancers in women: a systematic review and meta-analysis of 61 articles. Cancer Epidemiol Biomarkers Prev 2018;27:25-40.

20. Moch H, Cubilla AL, Humphrey PA, Reuter VE, Ulbright TM. The 2016 WHO Classification of Tumours of the Urinary System and Male Genital Organs-part A: renal, penile, and testicular tumours. Eur Urol 2016;70:93-105.

21. Kahn HJ, Marks A. A new monoclonal antibody, D2-40, for detection of lymphatic invasion in primary tumors. Lab Invest 2002;82: 1255-7.

22. Noma D, Inamura K, Matsuura Y, Hirata Y, Nakajima T, Yamazaki $\mathrm{H}$, et al. Prognostic effect of lymphovascular invasion on TNM staging in stage I non-small-cell lung cancer. Clin Lung Cancer 2018;19:e109-22.

23. Ahmed Farag AF, Elbarmelgi MY, Azim HA, Abozeid AA, Mashhour AN. TNMF versus TNM in staging of colorectal cancer. Int J Surg 2016;27:147-50.

24. Graziosi L, Marino E, Cavazzoni E, Donini A. Prognostic value of the seventh AJCC/UICC TNM classification of non-cardia gastric cancer. World J Surg Oncol 2013;11:103.

25. Albers P, Siener R, Kliesch S, Weissbach L, Krege S, Sparwasser C, et al. Risk factors for relapse in clinical stage I nonseminomatous testicular germ cell tumors: results of the German Testicular Cancer Study Group Trial. J Clin Oncol 2003;21:1505-12.

26. Vassilakopoulou M, de la Motte Rouge T, Colin P, Ouzzane A, Khayat D, Dimopoulos MA, et al. Outcomes after adjuvant chemotherapy in the treatment of high-risk urothelial carcinoma of the upper urinary tract (UUT-UC): results from a large multicenter collaborative study. Cancer 2011;117:5500-8.

27. Hellenthal NJ, Shariat SF, Margulis V, Karakiewicz PI, Roscigno M, Bolenz C, et al. Adjuvant chemotherapy for high risk upper tract urothelial carcinoma: results from the Upper Tract Urothelial Carcinoma Collaboration. J Urol 2009;182:900-6.

28. Xylinas E, Necchi A; Young Academic Urologists Urothelial Carcinoma Group of the European Association of Urology. Re: effectiveness of adjuvant chemotherapy after radical nephroureterectomy for locally advanced and/or positive regional lymph node upper tract urothelial carcinoma. Eur Urol 2017;72:473-74. 
Wen Liu, et al.

29. Shirotake S, Kikuchi E, Tanaka N, Matsumoto K, Miyazaki Y, Kobayashi $\mathrm{H}$, et al. Impact of an adjuvant chemotherapeutic regimen on the clinical outcome in high risk patients with upper tract urothelial carcinoma: a Japanese multi-institution experience. J Urol 2015;193:1122-8. 